\title{
COMMUNICATION
}

\section{Cu-Based Turn-on Fluorescent Sensors for Cu-rich Amyloid $\beta$ Aggregates}

Received 00th January 20xx, Accepted 00th January 20xx

\author{
Yiran Huang, ${ }^{a}$ Liang Sun, ${ }^{a}$ and Liviu M. Mirica ${ }^{a}{ }^{*}$
}

Protein misfolding and metal dishomeostasis are two key pathological factors of Alzheimer's disease. Previous studies have showed that $\mathrm{Cu}$-mediated $A \beta$ aggregation pathways lead to formation of neurotoxic $A \beta$ oligomers. Herein, we reported a series of picolinic acid-based $\mathrm{Cu}$-activatable sensors, which can be used for the fluorescence imaging of $\mathrm{Cu}$-rich $A \beta$ aggregates.

The formation of extracellular amyloid plaques containing the amyloid $\beta(A \beta)$ peptide is one of pathological hallmark in the Alzheimer's Disease patients' brains. ${ }^{1}$ Remarkably high concentration of $\mathrm{Cu}$ and $\mathrm{Zn}$ have been found within the amyloid plaques in AD patients' brains. ${ }^{2,3}$ Several studies have explored the interactions of metals with monomeric $A \beta$ peptides and their correlation with amyloid plaques and reactive oxygen species formation. ${ }^{4-10}$ These studies show that $\mathrm{Cu}$ can slow down the aggregation of $A \beta_{42}$ and reduce the fibrilization to a large extent, and is considered that the copper ions can stabilize the $A \beta_{42}$ oligomer species. ${ }^{11}$ In this regard, novel molecules that can modulate the interaction of copper ions with the soluble $A \beta_{42}$ species and alleviate the neurotoxicity may serve as novel therapeutic agents for $A D .^{12-14}$

In addition to the development of new therapeutic agents, it is also highly important to detect the $C u$-induced $A \beta$ species in AD. A number of fluorescent sensors have been developed to probe biological copper fluxes. ${ }^{15-19}$ These reporters can achieve high selectivity and signal-to-noise responses for copper ion imaging from cellular to tissue to whole animal settings. ${ }^{15,}$, $20-22$ However, only very few of the probes were utilized in detecting labile copper pools in AD, ${ }^{23,24}$ even though countless of studies have shown the copper homeostasis were dramatically disrupted. In this regard, to understand and probe the $\mathrm{Cu}$ mediated $A \beta$ aggregation process, it is significantly crucial to develop $\mathrm{Cu}-\mathrm{A} \beta$ specific probes. Herein, we rationally designed

\footnotetext{
a. Department of Chemistry, University of Illinois at Urbana-Champaign, $600 \mathrm{~S}$. Mathews Avenue, Urbana, IL 61801, United States

Correspondence: mirica@illinois.edu (L.M.M.)

† Electronic supplementary information (ESI) available: Experimental procedures, spectroscopic data, and copies of NMR spectra.
}

and synthesized a series of $\mathrm{Cu}$-based activable sensors to detect the $\mathrm{Cu}-\mathrm{A} \beta$ species in vitro and ex vivo. By linking the picolinic ester moiety with the strong $A \beta$ binding fragments, the copper ions can rapidly catalyze the hydrolysis reaction of the ester bond to generate the high fluorescent $A \beta$ binding molecules in vitro. More interestingly, the $\mathrm{Cu}$-responsive sensors can also promptly react with $\mathrm{Cu}-\mathrm{A} \beta$ oligomers and fibrils, resulting in a significant fluorescence turn-on, and indicating that the probes are also able to detect $C u-A \beta$ species in vitro. To confirm the $A \beta$ binding specificity of the probes, 5xFAD brain sections were stained with the developed sensors. As expected, when the brain sections only stained with the compounds, the fluorescence images show that the sensor has poor ability to detect amyloid plaque, given the low fluorescence intensity. However, to mimic the Cu-rich environment in $A D$ brain, with the addition of excess amounts of $\mathrm{Cu}(\mathrm{II})$ to the solution, the fluorescence images clearly indicate that the Cu-responsive sensors were activated by $\mathrm{Cu}(\mathrm{II})$ and release the high fluorescent amyloid binding fluorophores which can specifically label the amyloid plaques on the 5 XFAD brain sections.

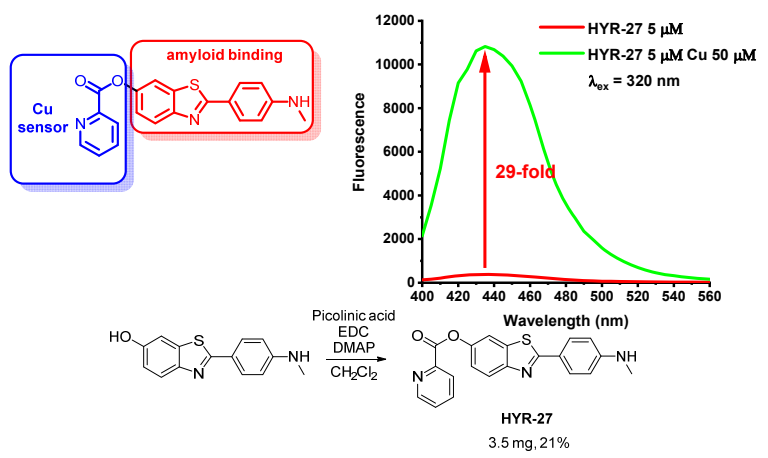

Fig. 1 Design and synthesis of Cu-based activatable sensor HYR-27. Cu(II) fluorescence turn-on effects of HYR-27. [HYR-27] = $5 \mu \mathrm{M}$, [Cu] $=50 \mu \mathrm{M}$ in PBS buffer $(\mathrm{pH}=7.4)$.

To detect $\mathrm{Cu}(\mathrm{II})$ in various $\mathrm{A} \beta$ species, the developed fluorescence probes includes two components: a $\mathrm{Cu}(\mathrm{II})-$ 
responsive 2-picolinic ester group that chelates $\mathrm{Cu}(\mathrm{II})$, activating the ester bond for hydrolysis, and a widely used $A \beta$ binding molecule, Pittsburgh compound B (PiB), which has high fluorescence intensity and can strongly interact with amyloid fibrils.

To evaluate the copper-responsive activity of the probe, we firstly performed the Cu turn-on assay in PBS buffer. With the attachment of the picolinic ester moiety, the fluorescence intensity of the probe is dramatically quenched. However, when we treated the $\mathrm{Cu}$ (II) with HYR-27, the fluorescence intensity significantly increased $\sim 29$ folds, indicating that the compounds can coordinates to the $\mathrm{Cu}$ (II) to facilitate the hydrolysis reaction to enhance the fluorescence intensity.
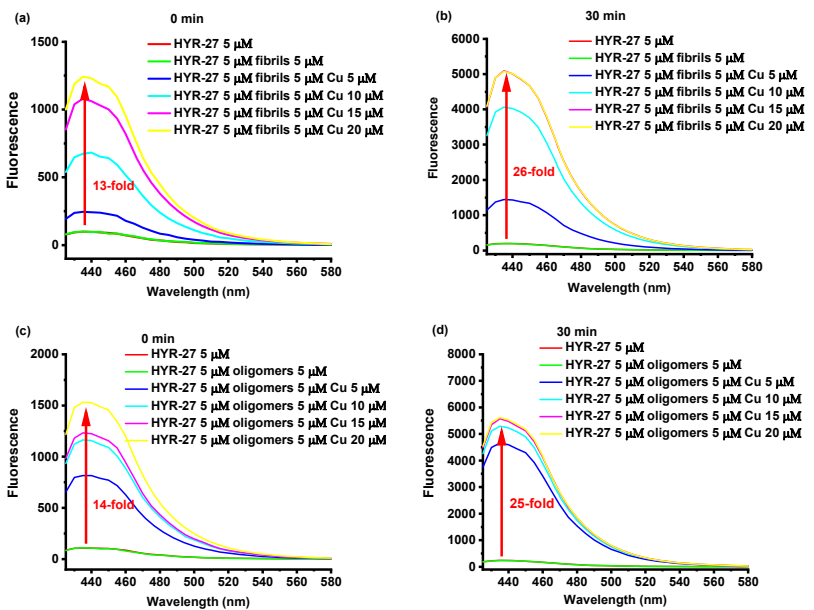

Fig. $2 \mathrm{Cu}$ (II) fluorescence turn-on results of HYR-27 toward $A \beta$ fibrils and oligomers. [HYR-27] $=5 \mu \mathrm{M},[\mathrm{AB}]=5 \mu \mathrm{M},[\mathrm{Cu}]=0-20 \mu \mathrm{M}$ in PBS buffer $(\mathrm{pH}=7.4)$.

Then, we explored the Cu-activable ability of HYR-27 with the presence of various $A \beta$ species. When the compound was treated with $A \beta$ fibrils only, no fluorescence enhancement was observed after 30 minutes incubation. However, when $\mathrm{Cu}-\mathrm{A} \beta$ fibrils were added to the solution, the fluorescence intensity dramatically increased, probably due to the rapid $\mathrm{Cu}(\mathrm{II})$ catalyzed hydrolysis reaction. With more copper ions adding into the solution, all HYR-27 were hydrolyzed to generate the $\mathrm{PiB}$ compounds, exhibiting a similar fluorescence turn-on effect ( 26 folds), comparing with the copper-responsive studies. Because $\mathrm{Cu}$ (II) can stabilize the formation of $A \beta$ oligomers, it is also highly crucial to investigate if the developed probe can also detect $\mathrm{Cu}-\mathrm{A} \beta$ oligomers. As a result, firstly, we treated the compounds with $A \beta$ oligomers only. Similar as $A \beta$ fibrils studies, no obvious fluorescence turn-on was observed with 30 minutes incubation. Excitingly, when $\mathrm{Cu}$ (II) was added to the solution, the fluorescence intensity immediately increased, indicating that the probe can compete with $A \beta$ oligomers to chelate the $\mathrm{Cu}(\mathrm{II})$ to facilitate the hydrolysis reaction to release the high fluorescent PiB probes. Furthermore, it also shows similar fluorescence enhancements, comparing with the control studies. Overall, the Cu-dependent fluorescence turn-on studies with or without various $A \beta$ species clearly demonstrate that the developed probes can be efficiently activated by the $\mathrm{Cu}(\mathrm{II})$, $\mathrm{Cu}(\mathrm{II})-\mathrm{A} \beta$ fibrils and oligomers, exhibiting appreciable fluorescence enhancement in vitro.

To further explore the $A \beta$ binding affinity and $\mathrm{Cu}$-activable activity of HYR-27, we also performed fluorescence staining studies with the probe on the 11-mon-old 5xFAD brain sections, which can rapidly develop severe amyloid pathology. Firstly, the brain sections were only stained with HYR-27. The low fluorescent image shows that the compound cannot efficiently detect amyloid plaques, which is consistent with the in vitro studies. To mimic the real Cu-rich environment in AD brain, we further performed the brain sections staining studies with the addition of $50 \mu \mathrm{M} \mathrm{Cu}(\mathrm{II})$. Excitingly, under same conditions, the fluorescence image clearly shows that the fluorescence intensity significantly increased after the incubation with $\mathrm{Cu}(\mathrm{II})$, indicating that HYR-27 was rapidly hydrolyzed to form PiB, which can strongly bind to the amyloid plaques, with the presence of the $\mathrm{Cu}(\mathrm{II})$.

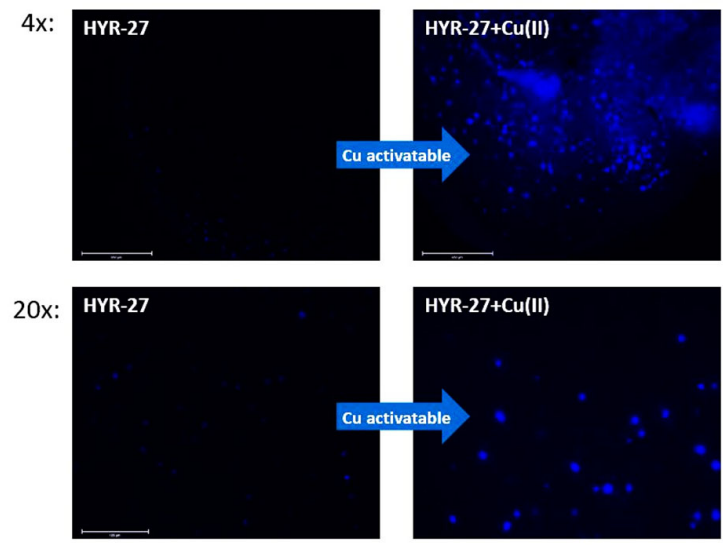

Fig. 3 Fluorescence microscopy images of 5xFAD mice brain sections incubated with compounds HYR-27 (left panel) and HYR-27+Cu (right panel). [HYR-27] = $5 \mu \mathrm{M},[\mathrm{Cu}]=50 \mu \mathrm{M}$. Scale bar (20x): $125 \mu \mathrm{m}$.

To further confirm the $A \beta$ binding specificity, the brain sections were firstly stained with HYR-27, HYR-27 with $\mathrm{Cu}(\mathrm{II})$ and sequentially immunostained with HJ3.4, which can bind to all $A \beta$ species. The fluorescence images show that HYR-27 did not have good colocalization with HJ3.4 antibody. However, with the presence of $\mathrm{Cu}(\mathrm{II})$, the hydrolyzed product of HYR-27 dramatically increases the colocalization with the pan- $A \beta$ antibody, exhibiting that the HYR-27 has high Cu-responsive ability which can be utilized to detect the $\mathrm{Cu}-\mathrm{A} \beta$ amyloid plaques on 5 XFAD brain sections. 

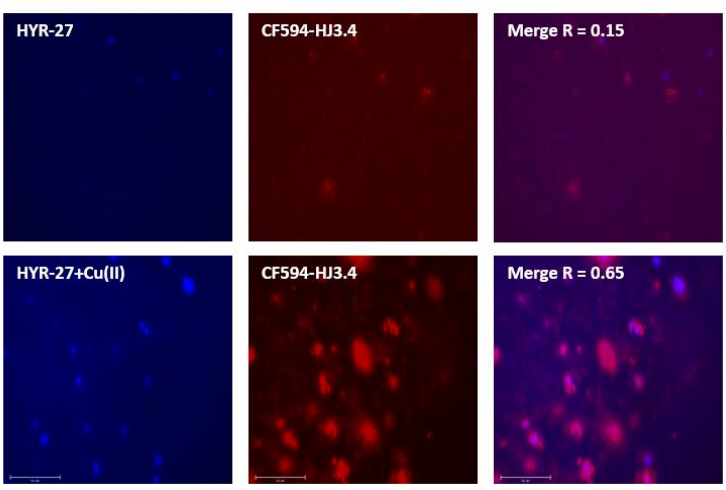

Fig. 4 Fluorescence microscopy images of 5xFAD mice brain sections incubated with compounds HYR-27 (top left panel), HYR-27+Cu (bottom left panel), HJ3.4 (middle panels), and merged images (right panel). [HYR-27] $=5 \mu \mathrm{M},[\mathrm{Cu}]=50 \mu \mathrm{M},[\mathrm{HJ} 3.4]=1 \mu \mathrm{g} / \mathrm{ml}$. Scale bar: $125 \mu \mathrm{m}$.

Since the chelation of $\mathrm{Cu}$ (II) can facilitate the hydrolysis of the picolinic ester bond, a strong $\mathrm{Cu}(\mathrm{II})$ binding ligand, $\mathrm{Me}_{2} \mathrm{HTACN}$, was introduced to the HYR-27 fragment. However, when $\mathrm{Cu}$ (II) was added, the fluorescence intensity was dramatically decreased, indicating that the copper ions only quenched the fluorescence intensity because of the paramagnetic nature, instead of catalyzing the hydrolysis reaction. The reason that $\mathrm{Cu}(\mathrm{II})$ was only served as a fluorescence quencher is probably that the TACN and pyridine ligand can easily chelate with $\mathrm{Cu}(\mathrm{II})$ to form 4-coodinates complex, blocking the binding site of the ester bond with copper ions, resulting in loss of esterase activity.
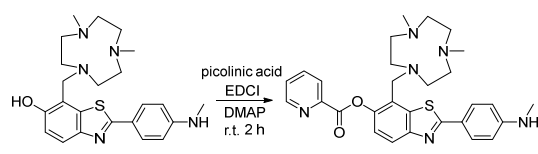

$10 \mathrm{mg}$

HYR-27-TACN

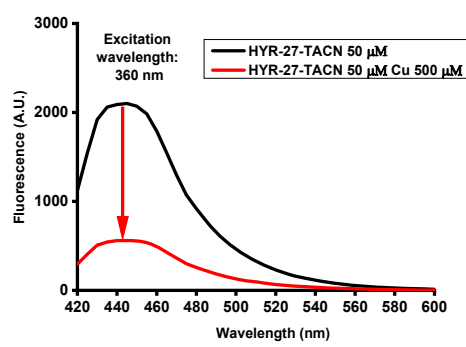

Fig. $5 \mathrm{Cu}(\mathrm{II})$ fluorescence turn-on effects of HYR-27-TACN. [HYR-27TACN $]=50 \mu \mathrm{M},[\mathrm{Cu}]=500 \mu \mathrm{M}$ in PBS buffer $(\mathrm{pH}=7.4)$.

After achieving promising results that HYR-27 can be activated by $\mathrm{Cu}(\mathrm{II})$, a series of curcumin derivatives which have high $A \beta$ binding affinity and near-infrared (NIR) emission properties were integrated with the $\mathrm{Cu}(\mathrm{II})$-responsive picolinic ester. The syntheses of the curcumin precursors, YL-1, -3, -5, were carried out by following previously reported procedures, ${ }^{25}$ and we synthesized the final $\mathrm{Cu}(\mathrm{II})$-responsive curcumin derivatives YL-2, -4, -6, via an EDC-mediated coupling reaction between 2-picolinic acid and the curcumin precursors.

Scheme 1 Synthesis of Curcumin Cu-based activatable sensors.

Step 1:

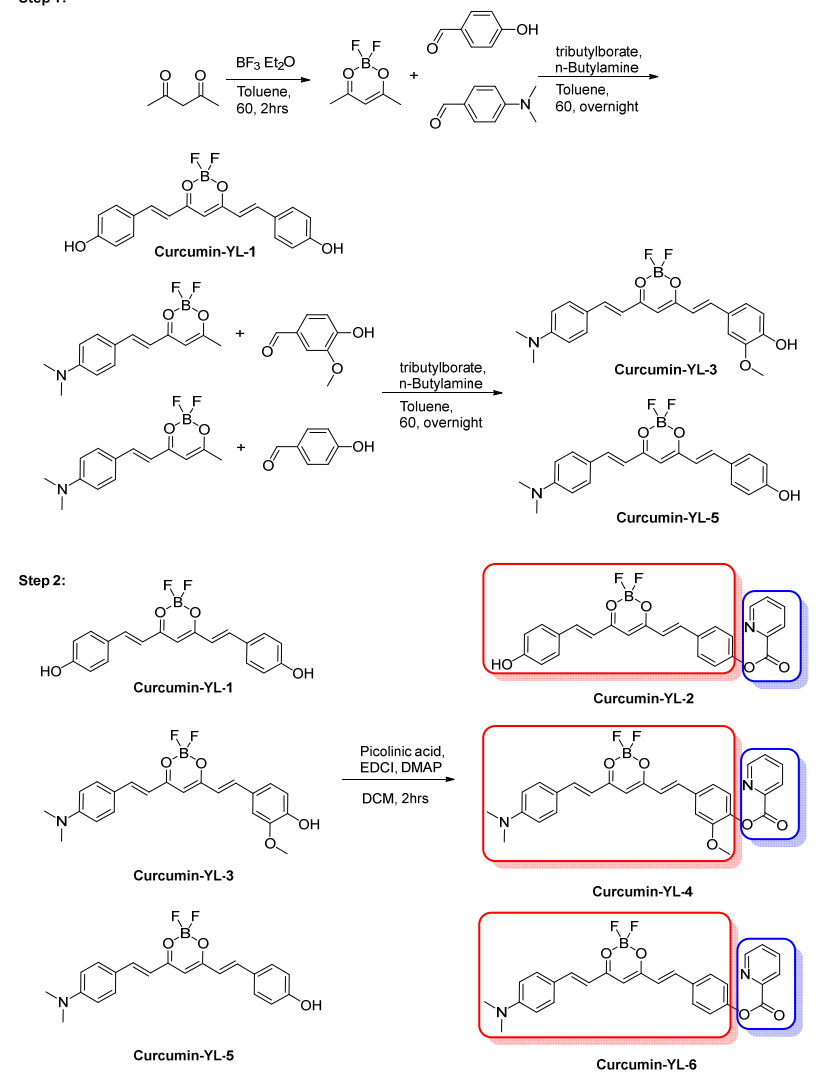

To confirm the Cu-activatable ability of the developed curcumin derivatives, the fluorescence turn-on experiments were performed in PBS buffer. However, the solubility of the compounds is poor in PBS buffer, so methanol was selected as the solvent to perform the $\mathrm{Cu}(\mathrm{II})$ turn-on assay. With the presence of $\mathrm{Cu}(\mathrm{II})$, all curcumin derivatives with the picolinic ester moiety have a remarkably fluorescence turn-on, proving that the developed compounds can be activated by the $\mathrm{Cu}(\mathrm{II})$ (Fig. 6).
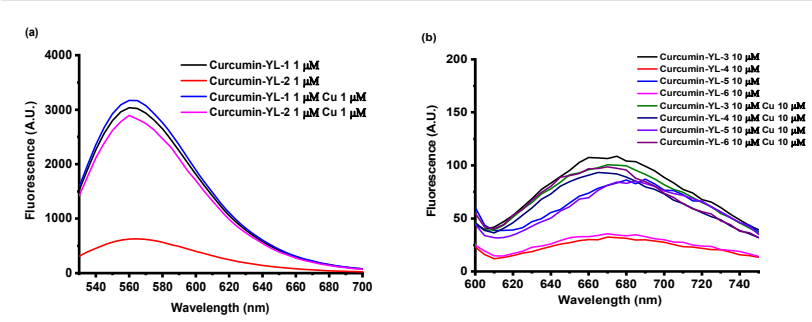

Fig. $6 \mathrm{Cu}(\mathrm{II})$ fluorescence turn-on effects of YL-1-6. [YL] $=1$ or $10 \mu \mathrm{M}$, $[\mathrm{Cu}]=1$ or $10 \mu \mathrm{M}$ in $\mathrm{MeOH}$. 
To investigate the Cu-activatable activity of the curcumin derivatives towards amyloid plaques, the fluorescence staining studies were also exploited with YL-4 on 5xFAD brain sections. We first stained the brain sections with YL-4 only, and the fluorescence images show that compound YL-4 is able to bind amyloid plaques on the brain sections (Fig. 7). However, the fluorescence intensity of the YL-4-stained amyloid plaques is low, and it also shows that the compound can only stain the mature fibrils where are the core of the amyloid plaques. By comparison, to mimic the $\mathrm{Cu}$-rich environment in $\mathrm{AD}$ brains, the 5XFAD brain sections were stained with YL-4 and $\mathrm{Cu}(\mathrm{II})$. Excitingly, with the addition of $\mathrm{Cu}(\mathrm{II})$, the fluorescence intensity of compound-stained amyloid plaques was dramatically increased, indicating that YL-4 was efficiently activated by $\mathrm{Cu}(\mathrm{II})$, generating the more fluorescent curcumin derivative, YL3. More interestingly, with the incubation of YL-4 and $\mathrm{Cu}(\mathrm{II})$, the resulting compound cannot only stain the core but also the peripheral region of the amyloid plaques where possibly $A \beta$ oligomers exist, exhibiting the potential ability to detect $\mathrm{Cu}-\mathrm{A} \beta$ oligomers of YL-4 ex vivo. For the other curcumin compounds, due to the poor solubility in PBS buffer, the brain sections staining studies were not performed. Overall, these brain section staining studies clearly demonstrate that YL-4 can be utilized as a Cu-responsive sensor for the detection of $\mathrm{Cu}$-rich amyloid plaques.
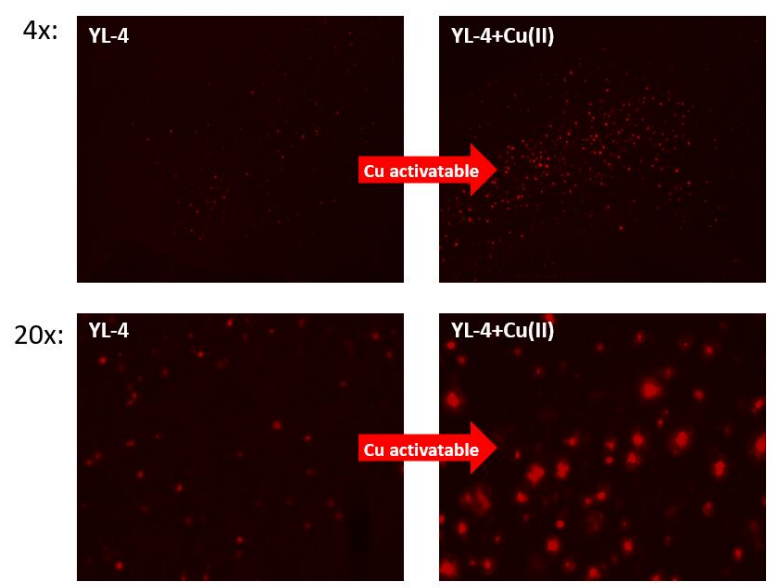

Fig. 7 Fluorescence microscopy images of 5xFAD mice brain sections incubated with compounds YL-4 (left panel) and YL-4+Cu (right panel). $[\mathrm{YL}-4]=50 \mu \mathrm{M},[\mathrm{Cu}]=50 \mu \mathrm{M}$. Scale bar: $125 \mu \mathrm{m}$.

In conclusion, we rationally designed and synthesized a series of Cu-based activatable sensors to detect the $\mathrm{Cu}-\mathrm{A} \beta$ species in vitro and ex vivo. With the introduction of the picolinate ester moiety to the strong $A \beta$-binding fragment, the developed sensors can efficiently chelate the $\mathrm{Cu}(\mathrm{II})$ to facilitate the hydrolysis of the ester bond to release the high fluorescent $A \beta$-binding molecules in vitro. Furthermore, the Cu-responsive sensors can also rapidly react with $\mathrm{Cu}-\mathrm{A} \beta$ oligomers and fibrils, resulting in a significant fluorescence turn-on, and indicating that the probes are also able to detect $\mathrm{Cu}-\mathrm{A} \beta$ species in vitro. To confirm the $A \beta$ binding specificity of the probes, brain section imaging studies were also performed with the compounds. As expected, if the brain sections were only stained with the compounds, the fluorescence images show that the sensor has poor ability to detect amyloid plaques, exhibiting low fluorescence intensity. However, to mimic the Cu-rich environment in $A D$ brain, excess $\mathrm{Cu}(\mathrm{II})$ was added to the incubation solution, and the resulting fluorescence images clearly demonstrate that the Cu-responsive sensors are activated by the copper ions and generate the highly fluorescent amyloid-binding fluorophores which can specifically label the amyloid plaques on the 5xFAD brain sections. Overall, the developed $\mathrm{Cu}$-based activatable sensors can be utilized to detect the $\mathrm{Cu}$-mediated $\mathrm{A} \beta$ species both in vitro and ex vivo.

\section{Conflicts of interest}

There are no conflicts to declare.

\section{ORCID}

Liviu M. Mirica: 0000-0003-0584-9508

Yiran Huang: 0000-0003-1755-9874

Liang Sun: 0000-0002-0080-0855

\section{References}

1. S. Tiwari, V. Atluri, A. Kaushik, A. Yndart and M. Nair, Int J Nanomed, 2019, 14, 5541-5554.

K. P. Kepp, Coord. Chem. Rev., 2017, 351, 127-159

K. P. Kepp, Chem. Rev., 2012, 112, 5193-5239.

C. J. Maynard, A. I. Bush, C. L. Masters, R. Cappai and Q. X. Li, International Journal of Experimental Pathology, 2005, 86, 147-159.

5. M. A. Smith, G. Perry, P. L. Richey, L. M. Sayre, V. E. Anderson, M. F. Beal and N. Kowall, Nature, 1996, 382, 120-121.

6. D. P. Smith, G. D. Ciccotosto, D. J. Tew, M. T. Fodero-Tavoletti, T. Johanssen, C. L. Masters, K. J. Barnham and R. Cappai, Biochemistry, 2007, 46, 2881-2891.

7. B. Alies, C. Bijani, S. Sayen, E. Guillon, P. Faller and C. Hureau, Inorg. Chem., 2012, 51, 12988-13000.

8. J. T. Pedersen, J. Ostergaard, N. Rozlosnik, B. Gammelgaard and N. H. Heegaard, J Biol Chem, 2011, 286, 26952-26963.

9. S. Bagheri, R. Squitti, T. Haertle, M. Siotto and A. A. Saboury, Front. Aging Neurosci., 2017, 9, 446.

10. G. z. Eskici and P. H. Axelsen, Biochemistry, 2012, 51, 6289-6311.

11. A. K. Sharma, S. T. Pavlova, J. Kim, J. Kim and L. M. Mirica, Metallomics, 2013, 5, 1529-1536.

12. C. Esmieu, D. Guettas, A. Conte-Daban, L. Sabater, P. Faller and C. Hureau, Inorg. Chem., 2019, 58, 13509-13527.

13. C. Hureau, J. Biol. Inorg. Chem., 2017, 22, S230-S230.

14. H. J. Yu, W. Zhao, Y. Zhou, G. J. Cheng, M. Sun, L. Wang, L. Yu, S. H. Liang and C. Z. Ran, Anal. Chim. Acta, 2020, 1097, 144-152.

15. T. Hirayama, G. C. Van de Bittner, L. W. Gray, S. Lutsenko and C. J. Chang, Proc. Natl. Acad. Sci. U. S. A., 2012, 109, 2228-2233.

16. S. Lee, C. Y. S. Chung, P. Liu, L. Craciun, Y. Nishikawa, K. J. Bruemmer, I. Hamachi, K. Saijo, E. W. Miller and C. J. Chang, J. Am. Chem. Soc., 2020, 142, 14993-15003.

17. K. J. Bruemmer, S. W. M. Crossley and C. J. Chang, Angew. Chem., Int. Ed., 2020, 59, 13734-13762.

18. D. A. lovan, S. Jia and C. J. Chang, Inorg. Chem., 2019, 58, 13546-13560.

19. H. Li, P. Zhang, L. P. Smaga, R. A. Hoffman and J. Chan, J. Am. Chem. Soc., 2015, 137, 15628-15631.

20. M. Saleem, M. Rafiq, M. Hanif, M. A. Shaheen and S. Y. Seo, J. Fluoresc., 2018, 28, 97-165.

21. L. Zeng, E. W. Miller, A. Pralle, E. Y. Isacoff and C. J. Chang, J. Am. Chem. Soc., 2006, 128, 10-11.

22. D. W. Domaille, L. Zeng and C. J. Chang, J. Am. Chem. Soc., 2010, 132, 1194-1195.

23. B. Muthuraj, S. Layek, S. N. Balaji, V. Trivedi and P. K. Iyer, ACS Chem. Neurosci., 2015, 6, 1880-1891.

24. Y. Y. Yu, P. Wang, X. D. Zhu, Q. W. Peng, Y. Zhou, T. X. Yin, Y. X. Liang and X. X. Yin, Analyst, 2018, 143, 323-331.

25. K. Liu, J. M. Chen, J. Chojnacki and S. J. Zhang, Tetrahedron Lett., 2013, 54, 2070 2073. 
TOC Graphic
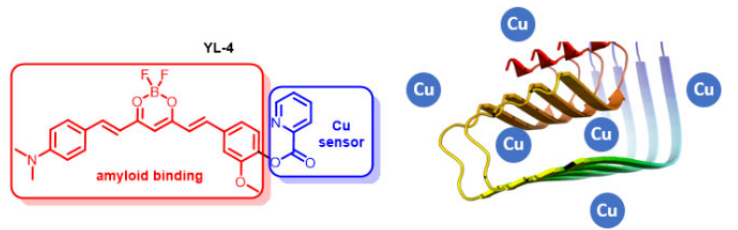

YL-4

$\mathrm{YL}-4+\mathrm{Cu}(\mathrm{II})$

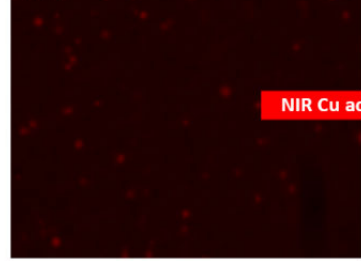

\section{Y.}

-

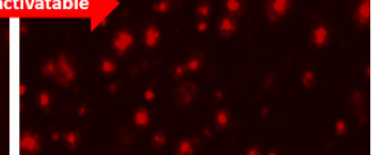

